\title{
The preventive effect of Chinese herbal preparation Xuebijing against hyperactive inflammation after hepato-pancreato-biliary surgery
}

\author{
Qifan Zhang ${ }^{1 \#}$, Jia Li ${ }^{2 \#}$, Xiaolu Liang ${ }^{1}$, Haorong Xie ${ }^{1}$, Hang Sun ${ }^{1}$, Xinxin Lin ${ }^{3}$, Jie Zhou ${ }^{1}$, Xiangjun $\mathrm{He}^{2}$, \\ Bili Zhu ${ }^{2}$
}

${ }^{1}$ Department of Hepatobiliary Surgery, ${ }^{2}$ Department of Huiqiao Building, Nanfang Hospital, Southern Medical University, Guangzhou 510515, China; ${ }^{3}$ The First Clinical College, Southern Medical University, Guangzhou 510515, China

Contributions: (I) Conception and design: Q Zhang, J Li; (II) Administrative support: X He, B Zhu; (III) Provision of study materials or patients: J Zhou; (IV) Collection and assembly of data: X Liang, H Xie, H Sun, X Lin; (V) Data analysis and interpretation: Q Zhang, J Li, X Liang, H Xie, H Sun; (VI) Manuscript writing: All authors; (VII) Final approval of manuscript: All authors.

\#These authors contributed equally to this work.

Correspondence to: Bili Zhu. Department of Huiqiao Building, Nanfang Hospital, Southern Medical University, No. 1023, South Shatai Road, Baiyun District, Guangzhou 510515, China. Email: zhubili361@163.com; bilizhunfyy@163.com.

Background: Hepato-pancreato-biliary (HPB) surgery is a primary treatment for benign and malignant diseases of the liver, biliary tract, and pancreas. Hyperactive inflammation has been indicated as a critical risk factor of post-operation death after HPB surgery. Xuebijing is an anti-inflammatory intravenous herbal preparation made from traditional Chinese medicines. Emerging evidence has implicated a protective role of Xuebijing against hyperactive inflammation.

Methods: A retrospective cohort study was conducted. We analyzed a total of 638 cases of HPB surgery, including hepatectomy, Whipple's surgery, and surgeries for cholelithiasis, which were divided into a Xuebijing treatment group and a conventional treatment group according to whether they were treated with Xuebijing injection or not. Clinical data related to liver function and inflammation were compared between the two groups after operation, including liver function index, white blood cell (WBC) count, neutrophil percentage (NE\%), C-reactive protein (CRP), serum interleukin-6 (IL-6), body temperature, mortality, incidence of adverse reaction, length of postoperative hospital stay, and hospitalization cost.

Results: Xuebijing injection was found to decrease the levels of inflammatory markers in the blood significantly, including WBC, NE\%, CRP, IL-6, and reduce the incidence of postoperative fever without prolonging in-hospital length or increasing cost compared to the conventional treatment group. Moreover, our data demonstrated that Xuebijing injection did not impact liver function after hepatectomy.

Conclusions: These results suggest that Xuebijing injection alleviates hyperactive inflammation caused by HPB surgery, and support the application of Xuebijing injection as a safe therapeutic approach against hyperactive inflammation in patients with HPB surgery.

Keywords: Hepato-pancreato-biliary surgery (HPB surgery); Xuebijing; hyperactive inflammation

Submitted May 14, 2019. Accepted for publication Jul 25, 2019.

doi: $10.21037 /$ atm.2019.07.78

View this article at: http://dx.doi.org/10.21037/atm.2019.07.78 


\section{Introduction}

Hepato-pancreato-biliary (HPB) surgery is one of the primary treatments for benign and malignant diseases of the liver, biliary tract, and pancreas $(1,2)$. However, due to the complexity of the regional anatomy, intraoperative tissue damage $(1,2)$ and gut microbial translocation (3) are nearly inevitable after HPB surgery. Therefore, systemic inflammation triggered by damaged tissue and bacterial translocation is a common complication after HPB surgery. Hyperactive inflammation that is aggravated from normal immune response to induce massive tissue injury may eventually cause systemic inflammatory response syndrome (SIRS) and multiple organ dysfunction syndrome (MODS) $(4,5)$. Indeed, the emerging evidence has implicated hyperactive inflammation as a risk factor for postoperative complications and mortality after HPB surgery (6-10).

The pathogenic mechanism of hyperactive inflammation after HPB surgery has not yet been well studied. Damaged tissue and bacterial translocation during HPB surgery are considered as the main factors triggering the immune response $(3,11)$. The massive tissue damage that frequently occurs in hemihepatectomy and Whipple's surgery is critically involved in inducing hyperactive inflammation by upregulating proinflammatory cytokines and enhancing oxidative stress. Currently, effective therapy for hyperactive inflammation after HPB surgery is still lacking.

Anti-inflammation and antioxidative-stress treatments are considered to be the primary strategies for managing hyperactive inflammation (4). Xuebijing is an intravenous herbal preparation made from five traditional Chinese medicines: Chishao (Radix Paeoniae Rubra), Danggui (Radix Angelica Sinensis), Chuanxiong (Rhizoma Chuanxiong), Honghua (Flos Carthami), and Danshen (Radix Salviae Miltiorrhizae). Xuebijing injection, which has been widely used in China, has been shown to inhibit hyperactive inflammation, prevent oxidative stress, neutralize endotoxins, improve microcirculation (12-20). In clinical trials, Xuebijing was demonstrated to fine-tune immune response and alleviate SIRS and MODS in patients with sepsis $(13,17,20)$ and severe burns $(21)$. However, whether Xuebijing injection prevents hyperactive inflammation after HPB surgery remains unclear.

In this study, we investigated the efficiency of Xuebijing injection as a therapy for hyperactive inflammation after HPB surgery. Respectively, 426, 120, and 92 cases of hepatectomy, Whipple's surgery, and surgeries for cholelithiasis were analyzed retrospectively. We found that
Xuebijing injection significantly alleviates inflammation after HPB surgery, suggesting an important therapeutic effect of Xuebijing injection for hyperactive inflammation in patients after HPB surgery.

\section{Methods}

\section{Patient selection}

A total of 638 cases of HPB surgery occurring between January 2015 and September 2017 were analyzed, including hepatectomy, Whipple's surgery, and surgeries for cholelithiasis. The inclusion criteria were as follows: (I) aged 18-75 years; (II) treated with partial hepatectomy, Whipple's surgery, and surgery for cholelithiasis. The exclusion criteria were as follows: (I) presence of biliary obstruction; (II) preoperatively diagnosed with severe diseases of the heart, lung, kidney, or other organs, (III) data missing; (IV) previous abdominal surgery history; (V) unconscious patients; (VI) immunodeficiency. After the operation, 103, 60, and 46 cases were treated with Xuebijing injection, and 323, 60, and 46 cases were treated without Xuebijing injection in patients who underwent hepatectomy, Whipple's surgery, and surgeries for cholelithiasis, respectively. For patients who underwent hepatectomy, the Xuebijing treatment group and the control group were further matched with a 1:1 propensity score and 202 patients were ultimately included in the study. Patient characteristic data are shown in Tables 1-3.

\section{Xuebijing treatment}

A Xuebijing injection of $50 \mathrm{~mL}$ was administrated 2 hours after surgery, and the treatment $(50 \mathrm{~mL}$, bid) continued for 5 days.

\section{Clinical data}

The clinical data collected included (I) the following general data: age, basic disease, preoperative levels of aspartate aminotransferase (AST), alanine aminotransferase (ALT), total bilirubin (TBIL), white blood cells (WBCs), neutrophil percentage (NE\%), and C-reactive protein (CRP); (II) the surgical data collected including the following: surgical methods, operation duration, intraoperative blood loss; (III) the postoperative data collected including the following: pathological diagnosis, postoperative level ALT, AST, TBIL, WBC, NE\%, CRP and body temperature, hospitalization 
Table 1 Patient characteristics in the hepatectomy cohort

\begin{tabular}{|c|c|c|c|}
\hline Variables & Xuebijing group $(n=101)$ & Control group $(n=101)$ & $P$ value \\
\hline Male (n, \%) & $83(82.2 \%)$ & $87(86.1 \%)$ & 0.441 \\
\hline Hepatitis (n, \%) & $92(90.5 \%)$ & $92(90.5 \%)$ & 1.000 \\
\hline Cirrhosis (n, \%) & $77(76.2 \%)$ & $75(74.3 \%)$ & 0.744 \\
\hline Operation time (min, $\bar{x} \pm \mathrm{s})$ & $208.9 \pm 98.7$ & $203.9 \pm 71.1$ & 0.690 \\
\hline Hepatic inflow occlusion [min, M (r)] & $0[0-38]$ & $0[0-30]$ & 0.308 \\
\hline Extensive liver resection (n, \%) & $53(52.5 \%)$ & $52(51.5 \%)$ & 0.888 \\
\hline Intraoperative blood loss (mL, $\bar{x} \pm s)$ & $270.9 \pm 248.0$ & $237.7 \pm 228.4$ & 0.334 \\
\hline TBIL ( $\mu \mathrm{mol} / \mathrm{L}, \bar{x} \pm \mathrm{S})$ & $12.8 \pm 7.0$ & $13.0 \pm 6.2$ & 0.821 \\
\hline WBC $\left(\times 10^{9} / L, \bar{x} \pm s\right)$ & $6.4 \pm 1.9$ & $6.2 \pm 1.6$ & 0.501 \\
\hline $\mathrm{NE}(\%, \bar{x} \pm \mathrm{s})$ & $58.3 \pm 10.1$ & $58.6 \pm 12.9$ & 0.866 \\
\hline Diameter of tumor $(\mathrm{cm}, \bar{x} \pm \mathrm{s})$ & $5.9 \pm 3.7$ & $5.4 \pm 3.2$ & 0.444 \\
\hline Number of tumor [n, M (r)] & $1[1-4]$ & $1[1-4]$ & 0.514 \\
\hline Child-Pugh A (n, \%) & $94(93.1 \%)$ & $97(96.0 \%)$ & 0.352 \\
\hline
\end{tabular}

ALT, alanine aminotransferase; AST, aspartate aminotransferase; TBIL, total bilirubin; WBC, white blood cell; NE\%, neutrophil percentage.

Table 2 Patient characteristics in pancreaticoduodenectomy cohort

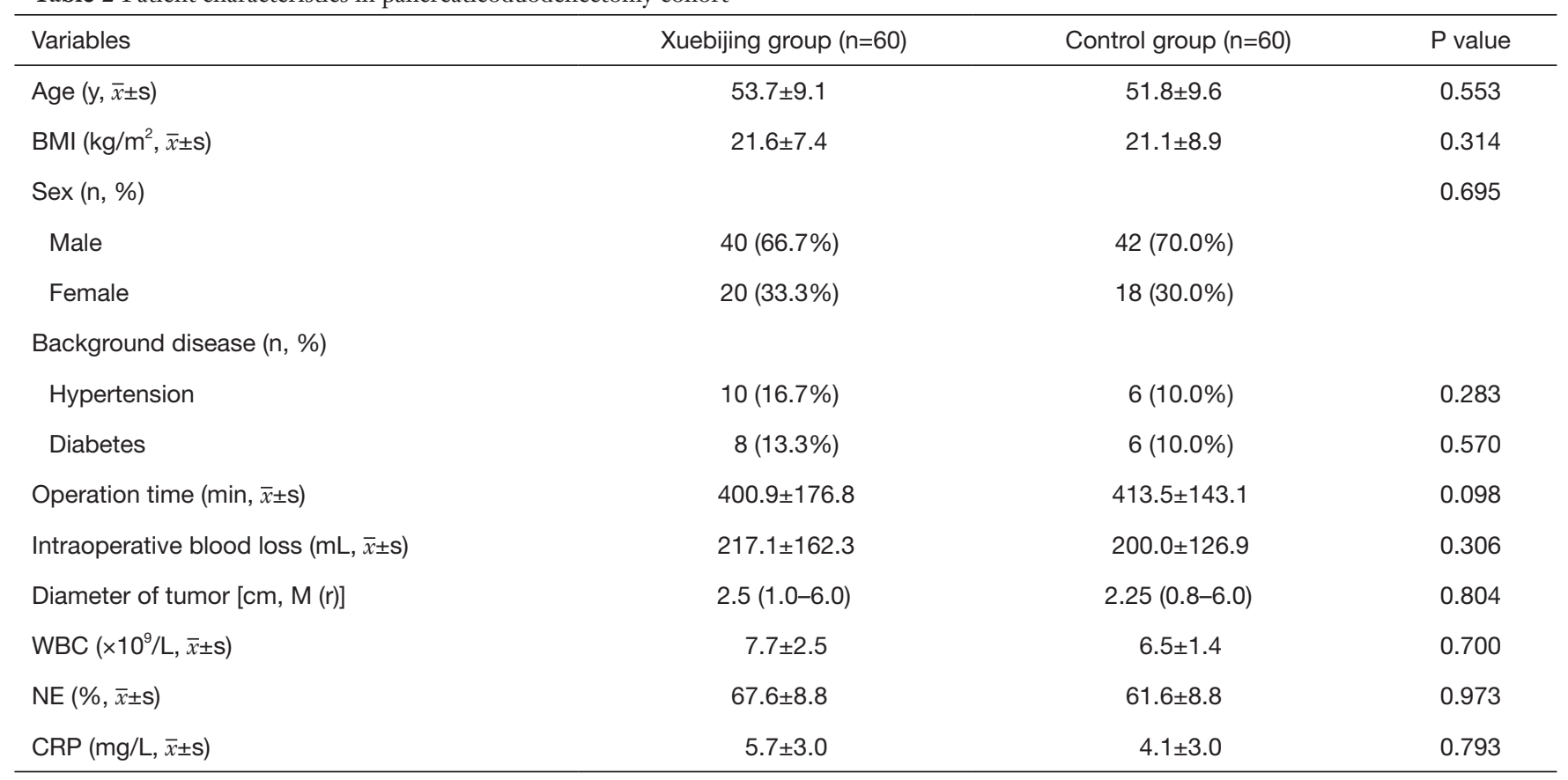

BMI, body mass index; WBC, white blood cell; NE\%, neutrophil percentage; CRP, C-reactive protein. 
Table 3 Patient characteristics in the cholelithiasis surgery cohort

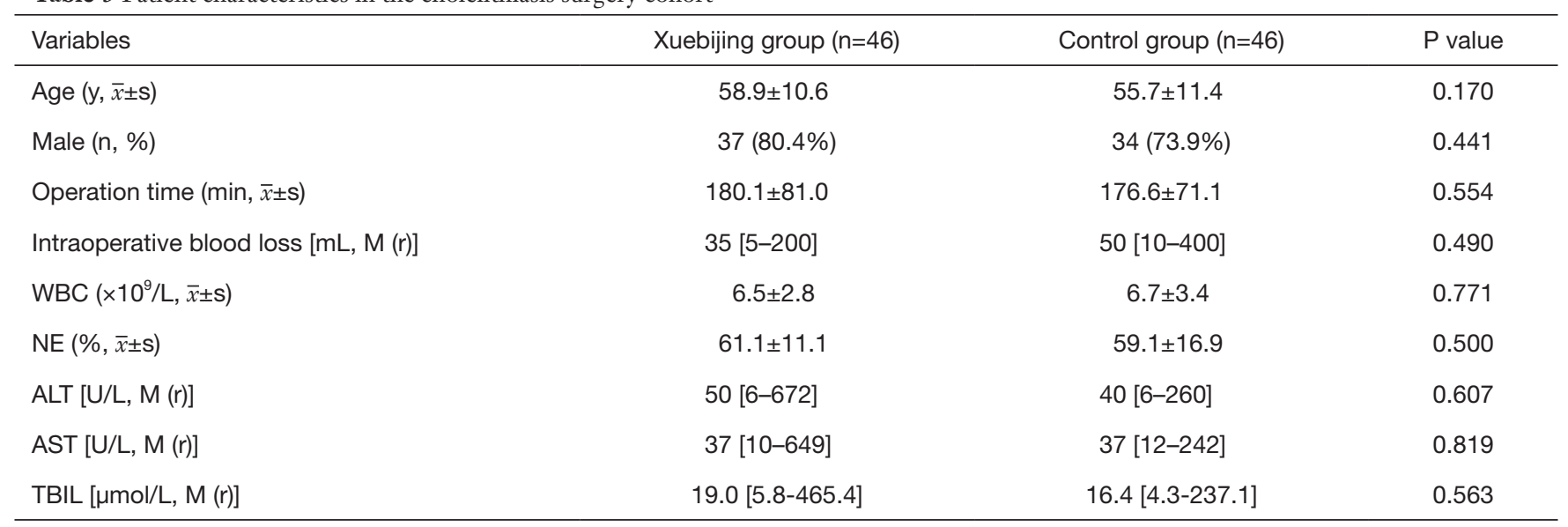

ALT, alanine aminotransferase; AST, aspartate aminotransferase; TBIL, total bilirubin; WBC, white blood cell; NE\%, neutrophil percentage.

cost, mortality, postoperative hospitalization days, and adverse drug reactions of Xuebijing.

\section{Blood samples and serum interleukin-6 (IL-6) measurement}

Blood samples were collected pre- and postoperatively at Nanfang Hospital Southern Medical University. All samples were collected in the morning before breakfast before surgery, and after surgery on postoperative day 3 at the hospital ward. Blood was drawn from the antebrachial vein into serum vacuum tubes. Serum samples were stored at $80^{\circ} \mathrm{C}$ until assayed with IL-6 enzyme-linked immunosorbent assay (ELISA) kit (Abcam; Cambridge, MA, USA).

\section{Statistical analysis}

Statistical Product and Service Solutions 20.0 software were used for data analysis. The data of normal distribution were analyzed using two independent samples $t$-test and presented as $\bar{x} \pm s$. Data of skew distribution were analyzed with the rank-sum test and presented as $M$ (range). The data between the two groups were compared using the chisquare test or Fisher's exact test. $\mathrm{P}<0.05$ was considered statistically significant.

\section{Results}

Xuebijing injection alleviates systemic inflammation without affecting liver function after bepatectomy

WBC, NE\%, and CRP levels were analyzed to determine if Xuebijing injection alleviates systemic inflammation in patients undergoing hepatectomy. After hepatectomy, both $\mathrm{WBC}$ and NE\% were increased on postoperative day 1 and started to recover on postoperative day 3 and 5 (Figure 1A,B). The CRP increased on postoperative day 1 and 3 and decreased on postoperative day 5 (Figure 1C). The CRP, WBC, and NE\% on postoperative day 3 and/or 5 were significantly different between the Xuebijing injection group and the control group; Xuebijing injection significantly decreased CRP, WBC, and NE\% on postoperative day 3 and/or 5 (Figure $1 A, B, C$ ). Levels of serum IL-6 were found to be significantly reduced by Xuebijing injection on postoperative day 3 (Figure 1D). The incidence of postoperative fever in Xuebijing group was significantly lower than that in the control group (Figure 1E). There was no significant difference in the length of postoperative hospital stay or hospitalization cost in the Xuebijing group compared with the control group (Figure 1F,G). Any symptoms that might be considered as side effects of Xuebijing injection were not found.

Liver function indices, including ALT, AST, and TBIL, were compared between the two groups to evaluate the effects of Xuebijing injection on postoperative liver dysfunction. The levels of ALT and AST in the two groups were significantly increased on postoperative day 1 and restored on postoperative day 3 and 5 (Figure 2). However, no significant difference in levels of ALT, AST, or TBIL was observed between the two groups (Figure 2). These results suggest that Xuebijing injection inhibits systemic inflammation without affecting liver function in patients after hepatectomy. 
A

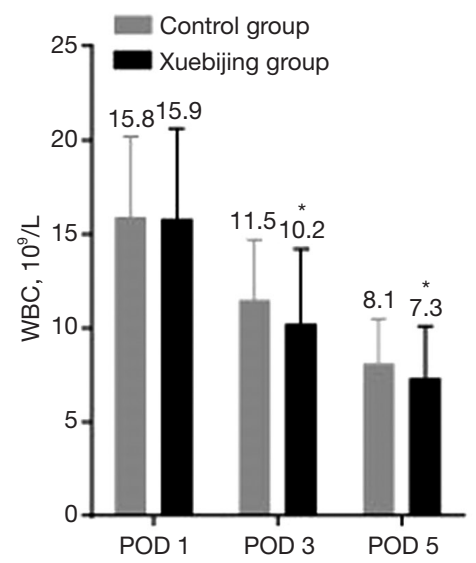

D

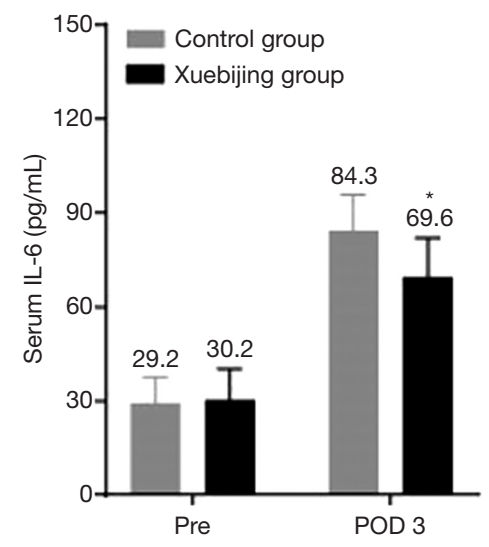

B
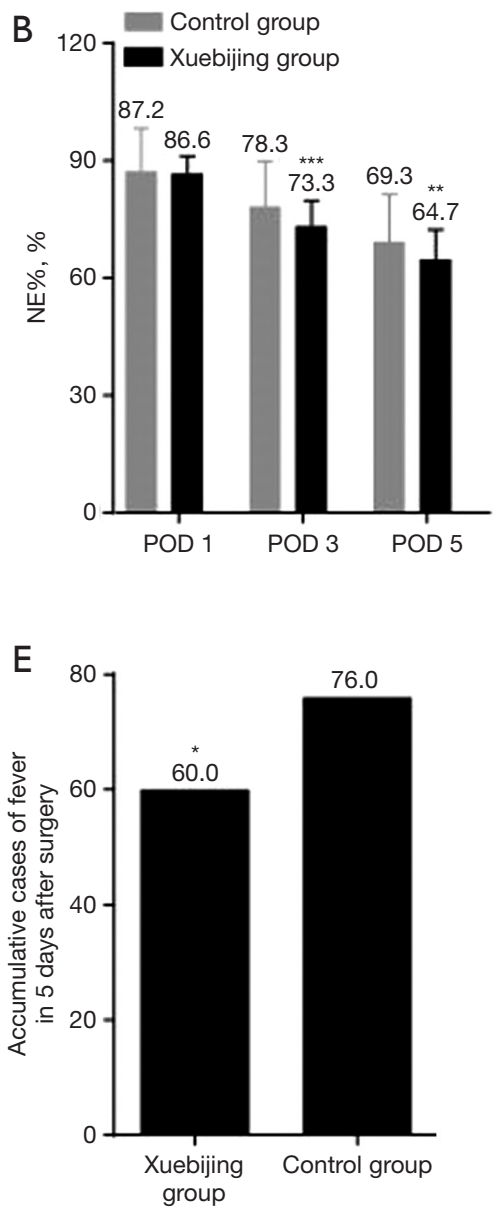

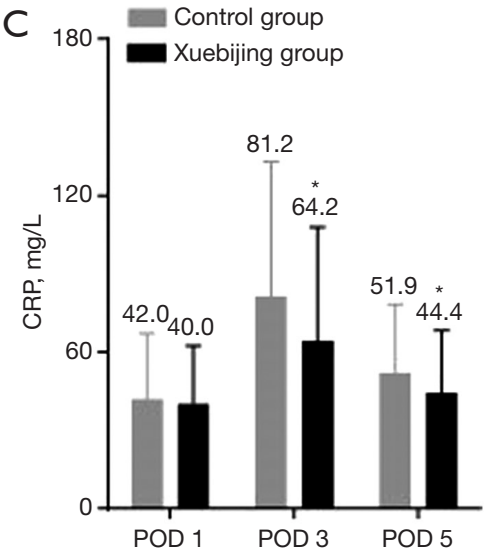

F

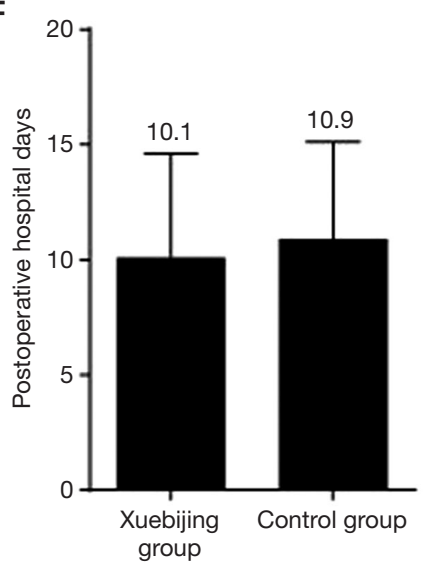

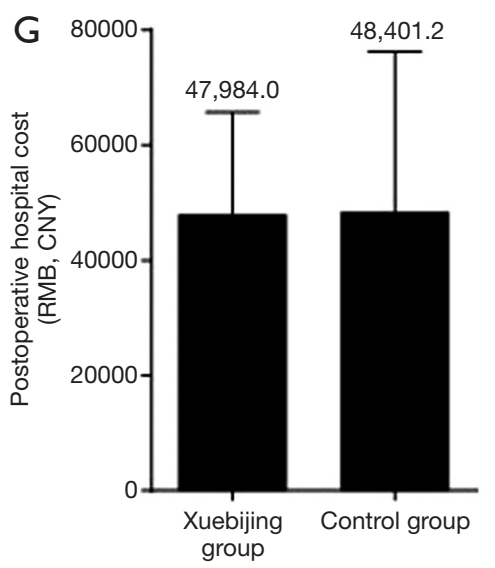

Figure 1 Xuebijing injection alleviates systemic inflammation in patients after hepatectomy. (A,B,C) Levels of WBC (A), NE\% (B), and CRP (C) in the Xuebijing injection group and control group. Xuebijing injection significantly lowered the levels of WBC, NE\%, and CRP at POD 3 and POD 5; (D) levels of serum IL-6 measured by ELISA; (E) cumulative cases of fever 5 days after hepatectomy. Xuebijing injection significantly prevented fever; $(\mathrm{F}, \mathrm{G})$ length of postoperative hospital stay and cost; no significant difference was observed between the two groups. Data in $\mathrm{A}, \mathrm{B}, \mathrm{C}, \mathrm{E}, \mathrm{F}$ and G represent mean $\pm \mathrm{SD} ; \mathrm{n}=101$. The mean value was displayed. Data in $\mathrm{D}$ represent mean $\pm \mathrm{SD} ; \mathrm{n}=10 .{ }^{*}, \mathrm{P}<0.05,{ }^{* *}, \mathrm{P}<0.01,{ }^{* * *}, \mathrm{P}<0.001$. WBC, white blood cell; NE\%, neutrophil percentage; CRP, C-reactive protein; IL-6, interleukin-6; ELISA, enzyme-linked immunosorbent assay; SD, standard deviation. 
Page 6 of 10

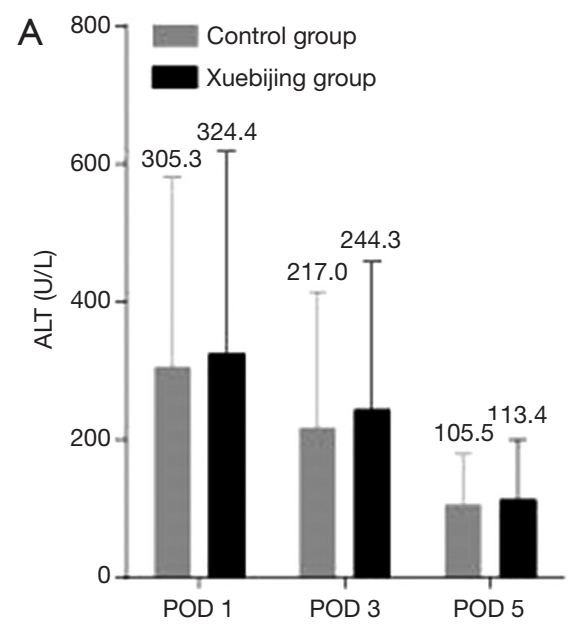

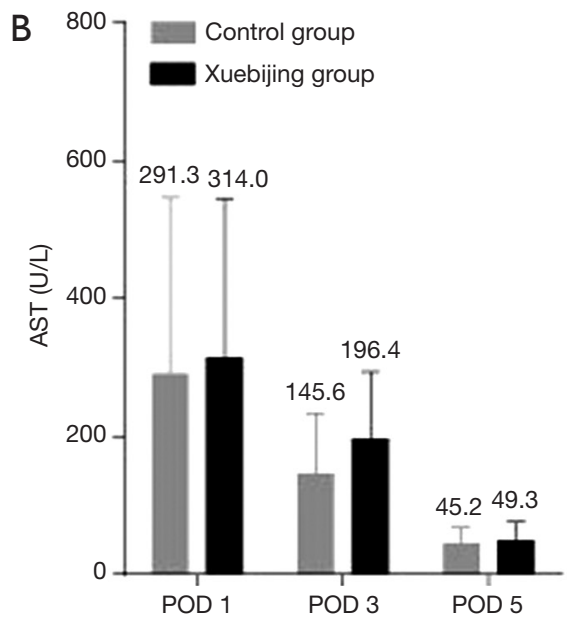

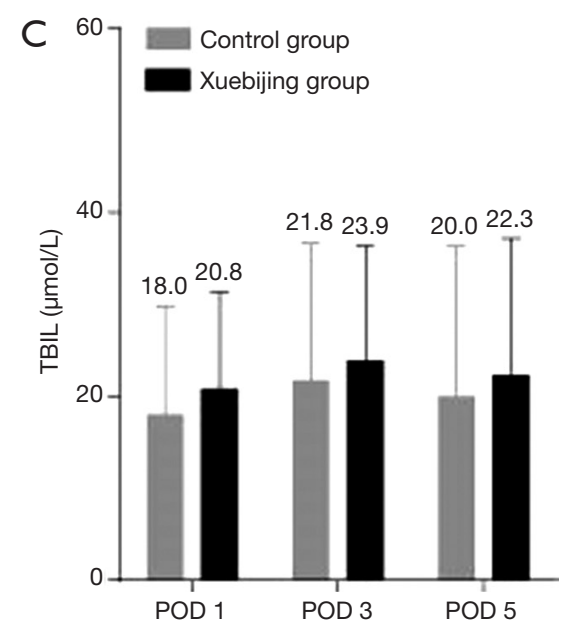

Figure 2 Xuebijing injection does not alter liver function after hepatectomy. Levels of ALT (A), AST (B), and TBIL (C) in the Xuebijing injection group and control group. No significant difference was observed between the two groups. Data represent mean \pm SD, $\mathrm{n}=101$. The mean value was displayed. ALT, alanine aminotransferase; AST, aspartate aminotransferase; TBIL, total bilirubin; SD, standard deviation.

\section{Xuebijing injection suppresses systemic inflammation after pancreaticoduodenectomy}

In patients who underwent Whipple's surgery, the levels of CRP, WBC, and NE\% in the two groups were increased on postoperative day 1 and recovered on postoperative day 3 and 5 (Figure $3 A, B, C$ ). The CRP, WBC, and $\mathrm{NE} \%$ on postoperative day 1 and 3 were significantly lower in the Xuebijing injection group (Figure $3 A, B, C$ ). Levels of serum IL-6 were found to be significantly decreased by Xuebijing injection on postoperative day 3 (Figure 3D). The incidence of postoperative fever in Xuebijing group was significantly lower than that in the control group (Figure 3E). There was no significant difference in postoperative hospital stay or hospitalization costs in the Xuebijing group compared to the control group (Figure 3F,G). No perioperative deaths or side effects of Xuebijing injection were observed in the two groups. These data demonstrate that Xuebijing injection suppresses systemic inflammation in patients after pancreaticoduodenectomy.

\section{Xuebijing injection inbibits systemic inflammation in patients with cholelithiasis after surgery}

In patients with cholelithiasis after surgery, levels of CPR, WBC, and NE\% were elevated on postoperative day 1 and started to normalize on postoperative day 3 and 5 (Figure $4 A, B, C$ ). The CRP, WBC, and $\mathrm{NE} \%$ on postoperative day 3 and 5 were significantly lower in the
Xuebijing injection group (Figure 4A,B,C). Serum IL-6 was found to be significantly decreased by Xuebijing injection on postoperative day 3 (Figure $4 D$ ). There was no significant difference in the incidence of postoperative fever (Figure 4E), postoperative hospital stay, or hospitalization costs (Figure 4F,G). No side effects were observed in the Xuebijing injection group. These data indicate that Xuebijing injection prevents systemic inflammation in patients with cholelithiasis after surgery.

\section{Discussion}

HPB surgery is considered as the most challenging abdominal surgery and has an extreme impact on the patient's physiology due to the complex procedures, large region of operation, long operation time, intraoperative tissue damage, and hyperactive inflammation after surgery. Although the development of surgical techniques and the improvement of surgical instruments have reduced the mortality of HPB surgery, the postoperative morbidity is still high $(1,2)$. Hyperactive inflammation, which may eventually cause SIRI or MODS, is associated with postoperative morbidity and mortality (4). Thus, clinical efforts to effectively inhibit hyperactive inflammation after HPB surgery are crucial for reducing both postoperative morbidity and mortality. In this study, we retrospectively analyzed the outcome of Xuebijing injection in patients after HPB surgery and found that Xuebijing injection significantly alleviates inflammation after HPB surgery, 

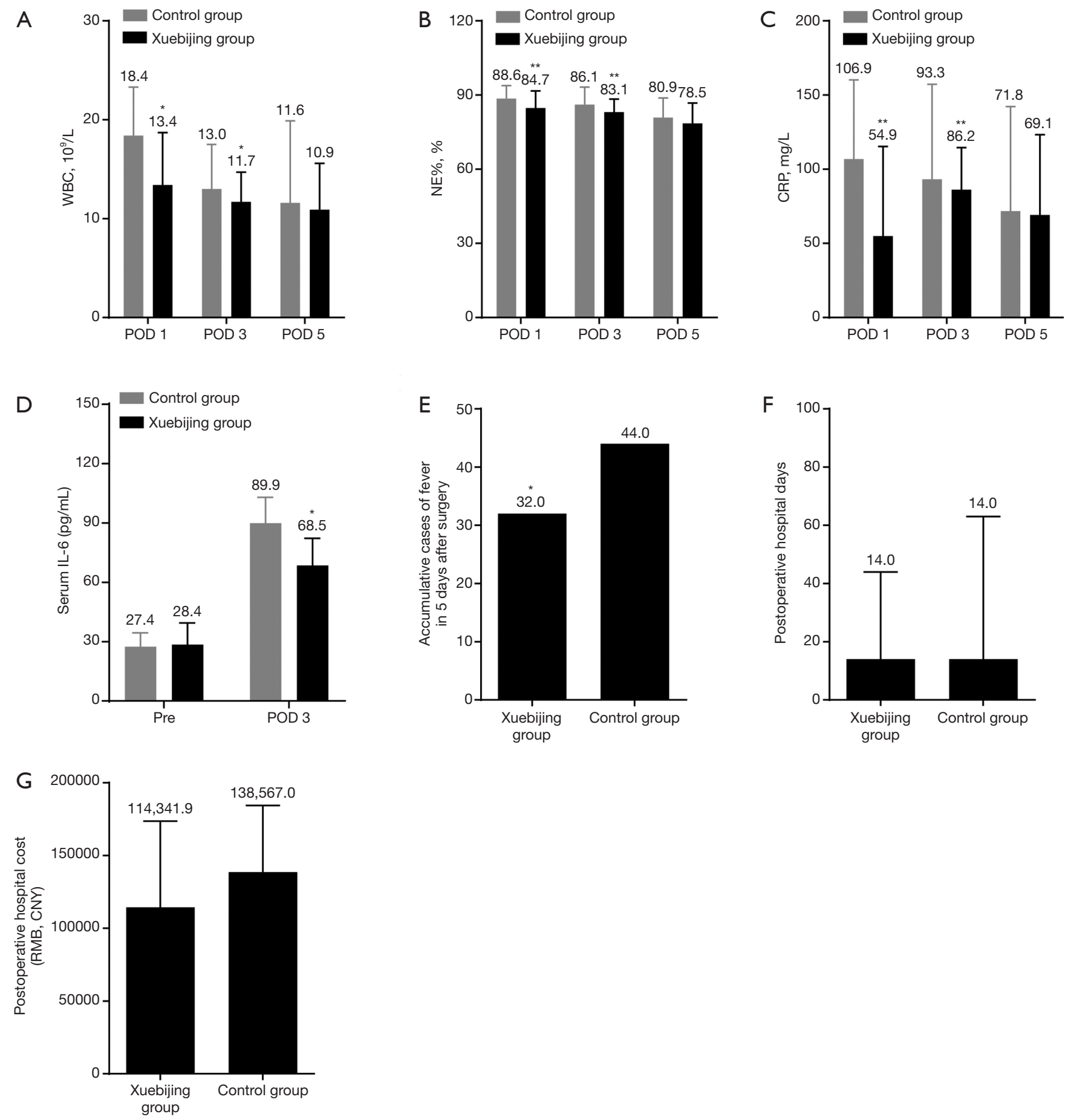

Figure 3 Xuebijing injection suppresses systemic inflammation after pancreaticoduodenectomy. (A,B,C) Levels of WBC (A), NE\% (B), and CRP (C) in the Xuebijing injection group and control group. Xuebijing injection significantly decreased the levels of WBC, NE\%, and CRP; (D) levels of serum IL-6 measured by ELISA; (E) cumulative cases of fever 5 days after pancreaticoduodenectomy. The incidence of postoperative fever was significantly lower in the Xuebijing group; (F,G) postoperative hospital days and cost; no significant difference was observed between the two groups. Data in A, B, C, E, F and G represent mean \pm SD; $\mathrm{n}=101$. The mean value was displayed. Data in $\mathrm{D}$ represent mean $\pm \mathrm{SD} ; \mathrm{n}=10$. * $\mathrm{P}<0.05,{ }^{* *}, \mathrm{P}<0.01,{ }^{* * *}, \mathrm{P}<0.001$. WBC, white blood cell; NE\%, neutrophil percentage; CRP, C-reactive protein; IL-6, interleukin-6; ELISA, enzyme-linked immunosorbent assay; SD, standard deviation. 

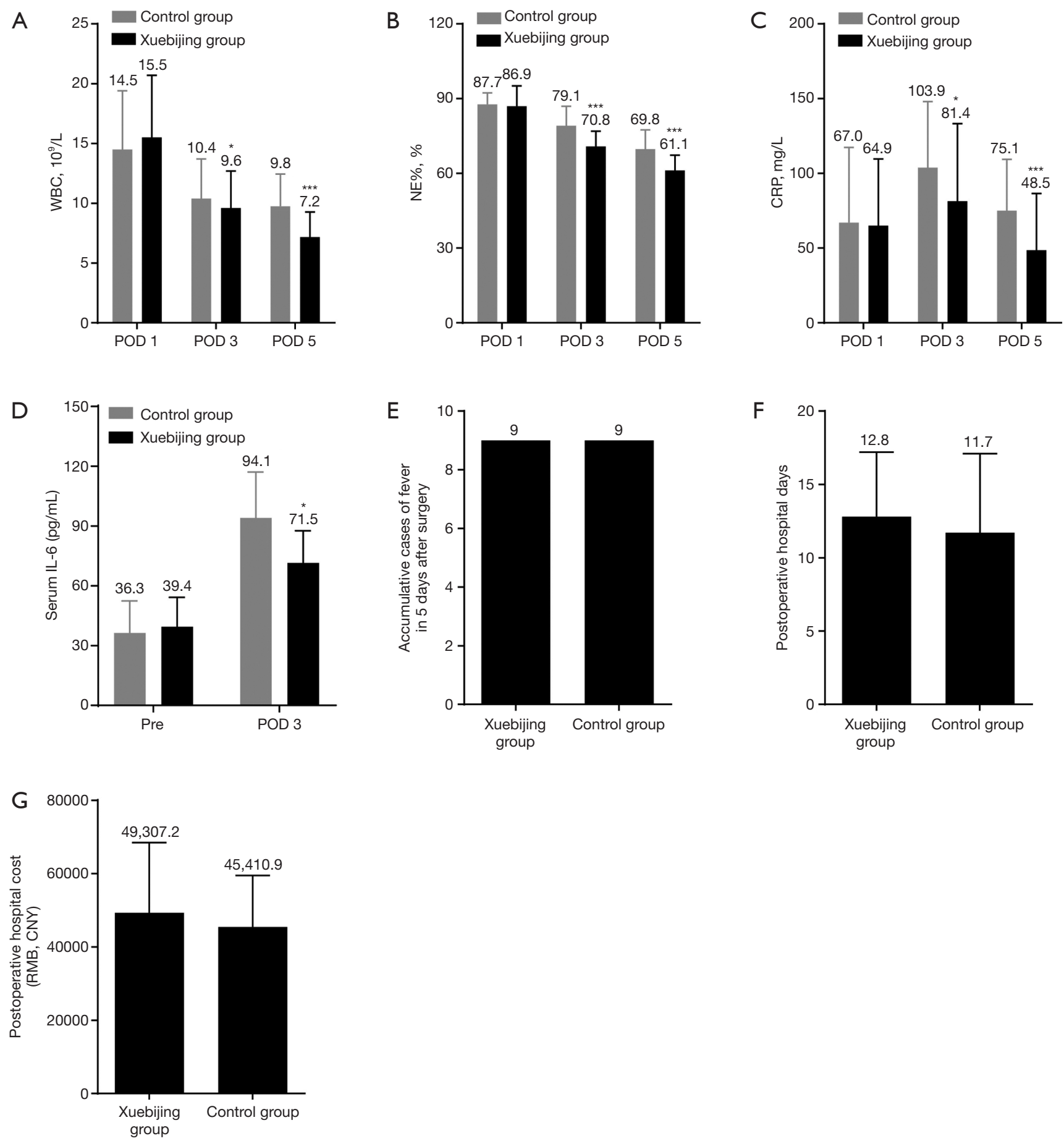

Figure 4 Xuebijing injection inhibits systemic inflammation in patients with cholelithiasis after surgery. (A,B,C) Levels of WBC (A), NE\% (B), and CRP (C) in the Xuebijing injection group and control group. Xuebijing injection significantly decreased the levels of WBC, NE\%, and CRP; (D) levels of serum IL-6 measured by ELISA; (E) cumulative cases of fever 5 days after surgery for cholelithiasis. No significant difference was observed between the two groups; $(\mathrm{F}, \mathrm{G})$ postoperative hospital days and cost; no significant difference was observed between two groups. Data in A, B, C, E, F and G represent mean \pm SD; $\mathrm{n}=101$. The mean value was displayed. Data in D represent mean \pm SD; $\mathrm{n}=10$. *, $\mathrm{P}<0.05$, ***, $\mathrm{P}<0.001$. WBC, white blood cell; NE\%, neutrophil percentage; CRP, C-reactive protein; IL-6, interleukin-6; ELISA, enzyme-linked immunosorbent assay; SD, standard deviation. 
including hepatectomy, Whipple's surgery, and surgeries for cholelithiasis. Our study highlights the protective effect of Xuebijing injection against hyperactive inflammation after HPB surgery.

Xuebijing injection is a confidential national technology product made from several traditional Chinese medicines. Xuebijing injection is widely used in China to treat hyperactive inflammation $(13,17,22)$. The mechanism by which Xuebijing injection inhibits hyperactive inflammation has been studied. Preliminary clinical studies have indicated that Xuebijing injection improves blood circulation to alleviate blood stasis and removes toxins $(19,20)$. Liu et al. reported that Xuebijing treatment attenuated inflammation by decreasing pro-inflammatory cytokines IL-6, IL-13, and TNF- $\alpha$, thus suppressing liver injury (23). Chen et al. also showed that Xuebijing treatment reduced oxidative stress and proinflammatory cytokines (24). Taken together, we can conclude that Xuebijing injection suppresses hyperactive inflammation after HPB surgery by possibly reducing proinflammatory cytokines, endotoxins, and oxidative stress.

Previous studies reported that Xuebijing injection has a hepatoprotective effect $(16,23,25)$. Liu et al. reported that Xuebijing treatment suppressed liver injury by decreasing pro-inflammatory cytokines IL-6, IL-13, and TNF- $\alpha$ (23). Liu et al. showed that Xuebijing injection potently inhibited apoptosis and IL-1 $\beta$ production in isolated hepatocytes in an NF- $\mathrm{kB}$-dependent manner, and also directly impacted hepatocyte inflammasome activation (16). Furthermore, a randomized controlled trial found that Xuebijing injection significantly decreased the levels of AST, ALT, and TBIL of patients in abdominal surgery (25). In our study on hepatectomy patients, compared with the control group, the Xuebijing group had lower levels of ALT, AST, and TBIL on postoperative day 1, 3, 5 but without a statistically significant difference. These resulted indicate that Xuebijing injection incurred no damage to liver function, and might have acted as a hepatoprotective treatment for the patients who underwent hepatectomy.

Several studies have reported the adverse effects caused by Xuebijing injection $(13,14,17,25)$. Chest tightness, dyspnea, pruritus, and palpitations were the most common adverse effects. In this study, all patients were cured without perioperative death. Only three patients developed pruritus, and they were healed after withdrawing Xuebijing injection. Moreover, there was no significant difference in postoperative hospital stay or hospitalization costs in the Xuebijing group compared with the control group. These findings suggest that Xuebijing injection appears to be safe and feasible in patients after HPB surgery.

In conclusion, our study reveals that Xuebijing injection has profound anti-inflammatory and potential hepatoprotective effects on HPB surgery and can be safely applied to prevent and treat excessive inflammatory reactions after HPB surgery.

\section{Acknowledgments}

Funding: This work was supported by the Guangzhou City Science and Technology Program 201400000001-3 and 262-1, the Guangdong Province Science and Technology Program 2017A030313684.

\section{Footnote}

Conflicts of Interest: The authors have no conflicts of interest to declare.

Ethical Statement: The authors are responsible for all aspects of the work in ensuring that questions related to the accuracy or integrity of any part of the work are appropriately investigated and resolved. This study gained approval from the Institutional Review Board of Nanfang Hospital, Southern Medical University, and all participants signed written informed consent (No. NFEC-2017-119).

\section{References}

1. Day RW, Brudvik KW, Vauthey JN, et al. Advances in hepatectomy technique: toward zero transfusions in the modern era of liver surgery. Surgery 2016;159:793-801.

2. Cai X. Laparoscopic liver resection: the current status and the future. Hepatobiliary Surg Nutr 2018;7:98-104.

3. Jeppsson B. Gut microbiota and surgical disease. Hepatobiliary Surg Nutr 2018;7:32-3.

4. Alazawi W, Pirmadjid N, Lahiri R, et al. Inflammatory and Immune Responses to Surgery and Their Clinical Impact. Ann Surg 2016;264:73-80.

5. Watt DG, Horgan PG, McMillan DC. Routine clinical markers of the magnitude of the systemic inflammatory response after elective operation: a systematic review. Surgery 2015;157:362-80.

6. Folch-Puy E. Importance of the liver in systemic complications associated with acute pancreatitis: the role of Kupffer cells. J Pathol 2007;211:383-8.

7. Lahiri R, Derwa Y, Bashir Z, et al. Systemic inflammatory response syndrome after major abdominal surgery 
predicted by early upregulation of TLR4 and TLR5. Ann Surg 2016;263:1028-37.

8. Schmidt SC, Hamann S, Langrehr JM, et al. Preoperative high-dose steroid administration attenuates the surgical stress response following liver resection: results of a prospective randomized study. J Hepatobiliary Pancreat Surg 2007;14:484-92.

9. Strey CW, Marquez-Pinilla RM, Markiewski MM, et al. Early post-operative measurement of cytokine plasma levels combined with pre-operative bilirubin levels identify high-risk patients after liver resection. Int J Mol Med 2011;27:447-54

10. Mao Y. Multiple disciplinary consensus on perioperative management of overwhelming inflammation for patients undergoing liver resection: an interpretation. Hepatobiliary Surg Nutr 2013;2:174-5.

11. Huang H, Tohme S, Al-Khafaji AB, et al. Damageassociated molecular pattern-activated neutrophil extracellular trap exacerbates sterile inflammatory liver injury. Hepatology 2015;62:600-14.

12. Cheng C, Lin JZ, Li L, et al. Pharmacokinetics and disposition of monoterpene glycosides derived from Paeonia lactiflora roots (Chishao) after intravenous dosing of antiseptic Xuebijing injection in human subjects and rats. Acta Pharmacol Sin 2016;37:530-44.

13. Gao J, Kong L, Liu S, et al. A prospective multicenter clinical study of Xuebijing injection in the treatment of sepsis and multiple organ dysfunction syndrome. Zhonghua Wei Zhong Bing Ji Jiu Yi Xue 2015;27:465-70.

14. He J, Tan Z, Zhang M, et al. Effect of Xuebijing injection on hemodynamics and endothelial function in patients with severe sepsis: a prospective study. Zhonghua Wei Zhong Bing Ji Jiu Yi Xue 2015;27:127-32.

15. Liu MW, Su MX, Zhang W, et al. Protective effect of Xuebijing injection on paraquat-induced pulmonary injury via down-regulating the expression of p38 MAPK in rats. BMC Complement Altern Med 2014;14:498.

16. Liu X, Hu Z, Zhou B, et al. Chinese herbal preparation Xuebijing potently inhibits inflammasome activation in hepatocytes and ameliorates mouse liver ischemia-

Cite this article as: Zhang Q, Li J, Liang X, Xie H, Sun H, Lin X, Zhou J, He X, Zhu B. The preventive effect of Chinese herbal preparation Xuebijing against hyperactive inflammation after hepato-pancreato-biliary surgery. Ann Transl Med 2019;7(18):481. doi: 10.21037/atm.2019.07.78 reperfusion injury. PLoS One 2015;10:e0131436.

17. Wang P, Song Y, Liu Z, et al. Xuebijing injection in the treatment of severe pneumonia: study protocol for a randomized controlled trial. Trials 2016;17:142.

18. Xu Q, Liu J, Guo X, et al. Xuebijing injection reduces organ injuries and improves survival by attenuating inflammatory responses and endothelial injury in heatstroke mice. BMC Complement Altern Med 2015;15:4.

19. Xu Q, Liu J, Wang Z, et al. Heat stress-induced disruption of endothelial barrier function is via PAR1 signaling and suppressed by Xuebijing injection. PLoS One 2015;10:e0118057.

20. Yin Q, Li C. Treatment effects of xuebijing injection in severe septic patients with disseminated intravascular coagulation. Evid Based Complement Alternat Med 2014;2014:949254.

21. Dai YL, Li JY, Bai HY, et al. Xuebijing Injection () increases early survival rate by alleviating pulmonary vasopermeability in rats subjected to severe burns. Chin J Integr Med 2017;23:703-8.

22. He F, Wang J, Liu Y, et al. Xuebijing injection induces anti-inflammatory-like effects and downregulates the expression of TLR4 and NF- $\kappa \mathrm{B}$ in lung injury caused by dichlorvos poisoning. Biomed Pharmacother 2018;106:1404-11.

23. Liu MW, Liu R, Wu HY, et al. Protective effect of Xuebijing injection on D-galactosamine- and lipopolysaccharide-induced acute liver injury in rats through the regulation of p38 MAPK, MMP-9 and HO-1 expression by increasing TIPE2 expression. Int J Mol Med 2016;38:1419-32.

24. Chen Y, Tong H, Pan Z, et al. Xuebijing injection attenuates pulmonary injury by reducing oxidative stress and proinflammatory damage in rats with heat stroke. Exp Ther Med 2017;13:3408-16.

25. Zhou X, Li H, Xie G, et al. Protective effect of the use of Xuebijing injection during laparotomy on perioperative inflammatory response and organ function. Zhonghua Wei Zhong Bing Ji Jiu Yi Xue 2014;26:258-63. 